\title{
MIME robotic device for upper-limb neurorehabilitation in subacute stroke subjects: A follow-up study
}

\author{
Peter S. Lum, PhD; ${ }^{1-2 *}$ Charles G. Burgar, MD; ${ }^{3}$ Machiel Van der Loos, PhD; ${ }^{4}$ Peggy C. Shor, OTR; ${ }^{4}$ \\ Matra Majmundar, OTR; ${ }^{4}$ Ruth Yap, MS $^{4}$ \\ ${ }^{1}$ Hunter Holmes McGuire Department of Veterans Affairs (VA) Medical Center, Richmond, VA; ${ }^{2}$ Biomedical Engineering, \\ The Catholic University of America, Washington, DC; ${ }^{3}$ Central Texas Veterans Health Care System, Temple, TX; ${ }^{4}$ VA Palo \\ Alto Health Care System, Palo Alto, CA
}

\begin{abstract}
This study presents results from a randomized controlled clinical trial of the Mirror Image Movement Enabler (MIME) robotic device for shoulder and elbow neurorehabilitation in subacute stroke patients, including data on the use of its bilateral training mode. MIME incorporates a PUMA 560 robot (Staubli Unimation Inc, Duncan, South Carolina) that applies forces to the paretic limb during unilateral and bilateral movements in three dimensions. Robot-assisted treatment (bilateral, unilateral, and combined bilateral and unilateral) was compared with conventional therapy. Similar to a previous study in chronic stroke, combined unilateral and bilateral robotic training had advantages compared with conventional therapy, producing larger improvements on a motor impairment scale and a measure of abnormal synergies. However, gains in all treatment groups were equivalent at the 6-month follow-up. Combined unilateral and bilateral training yielded functional gains that were similar to the gains from equivalent doses of unilateral-only robotic training, although the combined group had more hypertonia and less movement out of synergy at baseline. Robot-assisted treatment gains exceeded those expected from spontaneous recovery. These results are discussed in light of the need for further device development and continued clinical trials.
\end{abstract}

Key words: arm, bilateral, hemiparesis, movement, neurorehabilitation, rehabilitation, robotics, stroke, subacute, therapy, training.

\section{INTRODUCTION}

The development of robotic treatments is motivated by the need to improve clinical outcomes, the increase of public health burden associated with stroke-related disability [1], and the current emphasis on cost-reduction in healthcare [2]. Most stroke survivors receive one-on-one physical and occupational therapy for the resulting sensorimotor impairments. Neurorehabilitation of the upper limb is often abandoned early in favor of compensatory strategies. This decision is motivated by decreasing reimbursable patient-therapist contact time and the fact that the remaining intact limb with proper training and adaptive aids can perform most activities of daily living (ADL) involving the upper limbs. However, performing ADL one-handed is often cumbersome, increasing the time required and difficulty of the task, compared with performing ADL two-handed. These factors suggest that

\footnotetext{
Abbreviations: $\mathrm{ADL}=$ activities of daily living, $\mathrm{ARM}=$ Assisted Rehabilitation and Measurement (Guide), CI = constraint-induced, CVA = cerebrovascular accident, FIM = Functional Independence Measure, FM = Fugl-Meyer, $\max =$ maximum, MCID = minimally clinically important difference, MIME = Mirror Image Movement Enabler, MSS = Motor Status Score, NDT = NeuroDevelopmental Therapy, ROM = range of motion, RR\&D = Rehabilitation Research and Development, $\mathrm{VA}=$ Department of Veterans Affairs.

* Address all correspondence to Peter S. Lum, PhD; Biomedical Engineering, The Catholic University of America, Pangborn Hall, Room 131, 620 Michigan Avenue NE; Washington, DC 20064; 202-319-5657; fax: 202-319-4287. Email: lum@cua.edu
}

DOI: 10.1682/JRRD.2005.02.0044 
robotic devices can provide effective training for neurorehabilitation without increasing the burden on the clinicians or increasing the costs of healthcare. If commercially viable versions of these robotic devices can be developed, integration of robotic therapy into current practice could alleviate the labor-intensive aspects of neurorehabilitation and thereby increase the efficiency and effectiveness of therapists.

Another motivation for developing robotic treatments relates to the growing evidence that recovery from brain injury is heavily influenced by the sensorimotor experience following the injury [3]. An earlier seminal study showed that the highly repetitive task training of constraint-induced (CI) movement therapy, also known as CI therapy, can lead to gains in motor function [4]. More recent studies continue to confirm the positive effects of repetitive movement training on motor recovery after stroke [5]. While these studies focused on nonrobotic movement training, some evidence exists that robotic repetitive movement training might be even more effective [6], especially in moderately to severely impaired subjects who have difficulty performing unassisted repetitive movements. The rationale is that robots might enrich the sensorimotor experience by providing novel patient-environment interactions during active repetitive training. These novel training modes might eventually prove to be more effective than nonrobotic repetitive training. However, to date, we are aware of no study that has demonstrated this evidence.

The robotic device that has received the most clinical testing is the Massachusetts Institute of Technology (MIT)-Manus (Interactive Motion Technologies Inc, Cambridge, Massachusetts). A technical description was first presented in 1998 [7]. MIT-Manus is a 2 degrees-offreedom robot manipulator that assists shoulder and elbow movement by moving the patients hand in the horizontal plane. A novel impedance control mode allows MIT-Manus to be highly compliant when interacting with the patient's arm, thereby closely matching the human therapist-patient interaction. A series of clinical trials has shown that MIT-Manus provides effective treatment. Acute stroke subjects who received $25 \mathrm{~h}$ of MIT-Manus treatment had greater gains in motor function than control subjects who only received a placebo treatment [8-9]. Recent trials with chronic stroke subjects have demonstrated significant clinical gains with MIT-Manus training as well [10-12]. If this robotic treatment can be delivered cost-effectively, these results would justify use of MITManus as an adjunct to conventional treatment.

Another highly influential robotic device is the Assisted Rehabilitation and Measurement (ARM) Guide (Rehabilitation Institute of Chicago, University of California-Irvine) [13]. A motorized linear constraint provides active-assisted reaching movements in different directions. After 8 weeks of training in the ARM Guide, chronic stroke subjects had functional gains and improvements in reaching kinematics [14]. The most important result from this initial study was that the control group who received a matched amount of unassisted reaching movements had statistically identical gains. This emphasized the fact that highly repetitive active movements have therapeutic value and that the added value of assistance from a robot during active movements remained to be demonstrated. Motivated by these pioneering studies with MIT-Manus and ARM Guide, several less-tested approaches are under development [15-16].

\section{Our Past Work with MIME Device}

Over the last 10 years, with funding from the Department of Veterans Affairs (VA) Rehabilitation Research and Development (RR\&D) Service, we have investigated the use of robotics to facilitate upper-limb neurorehabilitation following stroke. The long-term goal is to provide patients with movement therapy that has been demonstrated to be equal or superior to what is currently available to patients. Notice that this framework differs from that of the MIT-Manus studies, where robotic training is compared with placebo controls. The motivation for our framework centers on the fact that robotic training is very different from conventional treatment, and it is unlikely that both types of treatment equally treat the range of impairments caused by stroke (hypertonia, weakness, abnormal synergies, etc). While the end goal is functional gain, direct comparison between robotic and conventional treatment allows one to identify which impairments are best treated by robotic therapy and which are best treated with conventional methods. This information would be a first step toward rational prescription of robotic therapy for treatment of specific impairments. The underlying premise is that robotic treatment may be more effective for some patients and less effective for others. Identifying these rules would lead to more effective integration of robotic training into conventional treatment. 
A series of prototypes led to the development in 1998 of the current MIME, which has several modes of robot-subject interactions [17-18] (Figure 1). The MIME unilateral modes were roughly patterned after the hands-on interactions that therapists use, and a novel bilateral mode was developed to assist mirror image movements. Early research recognized that bilateral exercises might provide advantages to similar unilateral movements [19-20], and a theoretical rationale for bilateral mirror image movements was developed [18]. Interestingly, two additional research groups have since

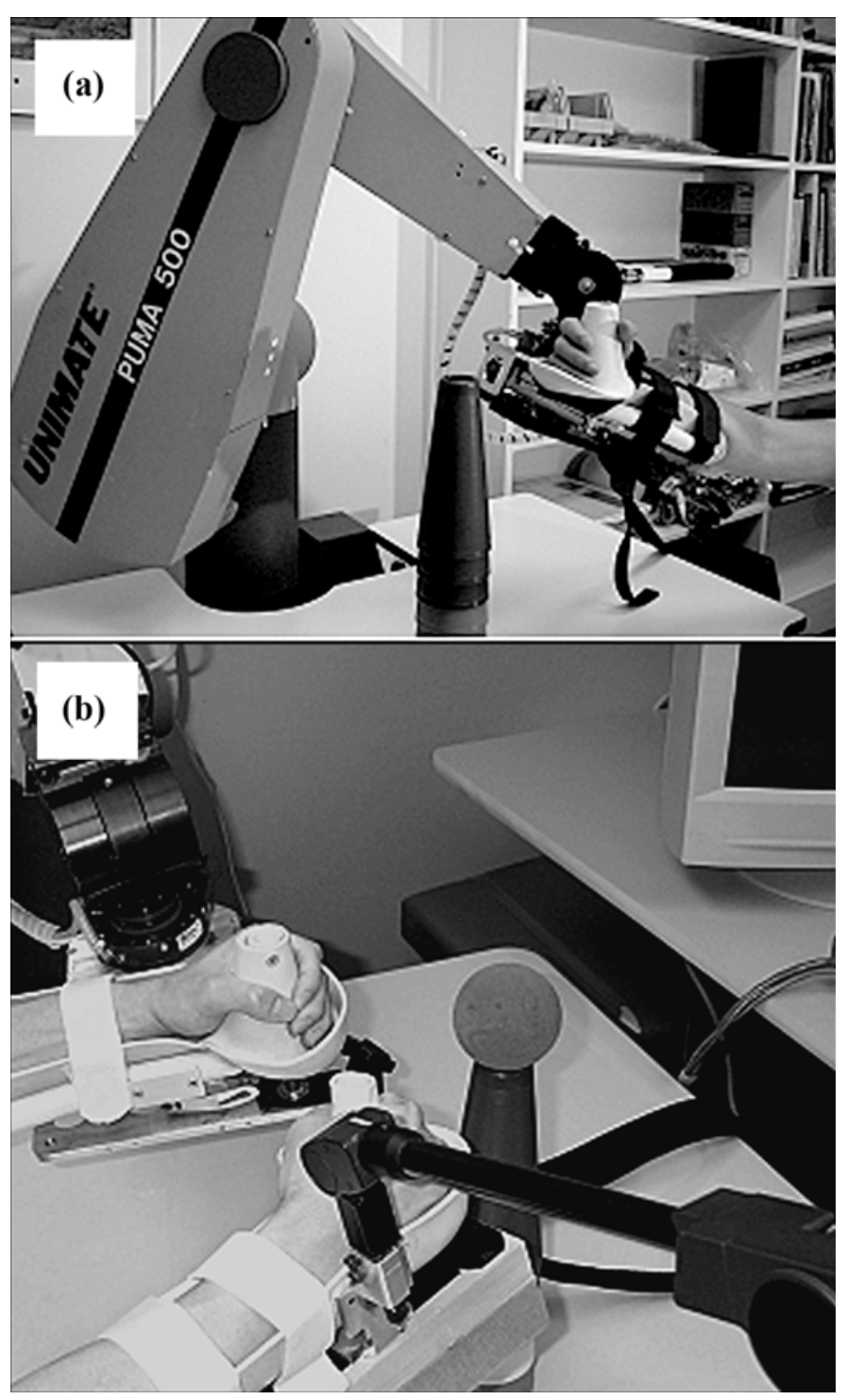

Figure 1.

Subjects performing (a) unilateral and (b) bilateral movements with Mirror Image Movement Enabler system. developed robotic devices for bilateral stroke therapy [21-22], and their preliminary results indicate the devices are effective.

\section{Description of MIME Device}

During our research with the MIME, subjects were seated in a wheelchair in front of a height-adjustable table. Straps and a contoured seat (Sunrise Medical, Carlsbad, California) limited torso movement, and the affected limb was strapped to a forearm splint that restricted wrist and hand movement. A robot manipulator (PUMA 560, Staubli Unimation Inc, Duncan, South Carolina) attached to the splint applied forces to the limb that a therapist normally provided. The 6 degrees of freedom of the robot allowed the forearm to be positioned within a large range of positions and orientations in three-dimensional space. The forces and torques between the robot and the affected limb were measured by a sixaxis sensor.

We used four modes of robot-assisted movement: passive, active-assisted, active-constrained, and bilateral. In passive mode, the subject relaxed as the robot moved the limb toward a target with a predetermined trajectory. In active-assisted mode, the subject triggered initiation of the movement with volitional force toward the target and "worked with the robot" as it moved the limb. In active-constrained mode, the robot provided a viscous resistance in the direction of the desired movement and spring-like restoring forces perpendicular to the movement direction as the subject attempted to reach toward the target with maximal effort. In bilateral mode, the subject attempted bilateral mirror image movements while the robot assisted the affected limb by continuously moving the affected forearm to the contralateral forearm's mirror image position and orientation. During bilateral mode, the two forearms were kept in mirror symmetry by a position digitizer (MicroScribe 3DL, Immersion Corp, San Jose, California), which measured the movement of the contralateral forearm and provided coordinates for the robot motion controller. The digitizer can measure arbitrary forearm trajectories with minimal resistance to movement (effective weight $<2 \mathrm{~N}$ ).

\section{Clinical Trial in Chronic Stroke}

Our initial clinical testing goal was to compare a therapy program of robotic manipulation with an equally intensive program of conventional therapy techniques [23]. During this trial, 27 chronic stroke subjects received 
24 one-hour sessions over 2 months. Subjects in the robot group practiced shoulder and elbow movements while assisted by MIME. Subjects in the control group received NeuroDevelopmental Therapy (NDT) [24] targeting proximal upper-limb function. We used the last $5 \mathrm{~min}$ of each session to expose the subject to the robot.

In the robot group, targeted reaching movements that started near the body and ended farther away were emphasized. Four point-to-point reaching directions were trained: forward-medial (shoulder flexion, adduction), directly forward (shoulder flexion), forward-lateral (shoulder flexion, abduction, external rotation), and directly lateral (abduction, external rotation). For each of these directions, targets could be located at tabletop, shoulder, or eye level. These 12 targeted reaching motions formed a core set of movements. Subjects practiced some or all of these movements in each session. Movements were kept within each subject's passive range of motion (ROM). During active-constrained movements, feedback was used to track and motivate performance. This feedback was either the fraction of the movement completed or the time to complete three repetitions. Time permitting, subjects practiced isolated elbow extension movements while assisted by MIME.

A typical control group session involved approximately 10 min of establishing a physical postural base of support and assessing and facilitating the alignment of the shoulder. Approximately $35 \mathrm{~min}$ was devoted to graded application of arm use via functional leisure and self-care tasks. Therapists emphasized the reeducation of muscles using a sensorimotor approach to control motor output. Subjects needed to show ability to independently perform basic mass functional movements before progressing to more isolated advanced functional patterns. They progressed within each movement by increasing the number of repetitions, weight of item being handled, height at which tasks were done, etc. In the last $10 \mathrm{~min}$, subjects practiced the highest level task that was completed, with additional review and assessment of the shoulder. Subjects received exposure to the robot for 5 min within each session, but the robot provided no active assistance. The robot provided a moving target, and subjects attempted to track the target with their hand or stack cones on top of the robot end effector as it moved. An NDT-certified therapist with 13 years experience in treating neurologically injured patients provided therapy. The therapist's feedback was consistent with a "conventional therapy" approach. The therapist provided continual comments on the quality of movement to ensure continued interest and facilitate success of the task. If the subject could not achieve a task, the therapist would suggest other ways of doing it, or if the therapist felt that further attempts at the task would lead to frustration, the level of difficulty would be decreased.

When compared with conventional treatment, robotic therapy had advantages in terms of clinical and biomechanical measures. The robot group had statistically larger improvements on the proximal Fugl-Meyer (FM) [25] after both 1 month and 2 months of treatment. No between-group differences were found in the distal FM. After 2 months of treatment, the robot group had significantly greater strength improvements in joint actions that received focused training (shoulder flexion, abduction, adduction, and elbow extension). We measured the kinematics of free reaching movements by attaching a lightweight, counterbalanced digitizer (Microscribe 3DL, Immersion Corp, San Jose, California) to the forearm. Analysis of these data found that the robot group had larger increases in reach extent in six of the eight movements tested. At the 6-month follow-up, the groups no longer differed in terms of the proximal FM scale. These results of this clinical trial are evidence that this regimented program of robot-assisted movement had advantages compared with an equally intensive program of conventional treatment, primarily in the rate of impairment reduction and in quantitative measures of movement and strength.

Further examination of the data collected during the robotic training provided evidence of improved muscle activation patterns [26]. Analysis was performed on the interaction forces, kinematics, and electromyograms recorded during training of eight different movement patterns in active-constrained mode. These movements were chosen because they were performed consistently in each session and performance feedback was provided to motivate maximal performance. Work output during the training was significantly increased by week 3 in five of the eight patterns; by week 5 , significant work gains were seen in all eight patterns. These gains were too early to have been because of muscle hypertrophy and were more likely because of increased neural activation of paretic muscles. Electromyographic data provided additional evidence: improved muscle activation patterns were observed in the four movement patterns that started at tabletop level and ended at shoulder level. In contrast, no evidence of improved muscle activation patterns was found in any of the tabletop movements, with increased activation of antagonists in two movement patterns. This 
dichotomy may have been because of compensation with shoulder girdle movement, which limited the effectiveness of the tabletop movements in promoting neurorehabilitation.

\section{Current Subacute Clinical Trial}

The goals of this study were to confirm the previous results in the clinical trial of chronic subjects and to identify the essential therapeutic features of MIME robotic therapy. In particular, the bilateral mode is unique to MIME, and the study was designed to evaluate the potential unique benefits of this mode. Our working hypothesis was that when the two modes are combined, the bilateral mode enhances the effects of the more conventional unilateral mode.

Why the bilateral mode would enhance the effectiveness of the unilateral mode relates to the hypothesis that the potential mechanisms that underlie the two modes are different. The unilateral mode targets corticospinal pathways from the contralateral damaged cortical hemisphere, while the bilateral mode involves the undamaged hemisphere. The bilateral mode may facilitate corticospinal ipsilateral pathways, cortical projections to brain stem pathways, or the damaged hemisphere through the corpus callosum. All or some of these pathways might contribute to motor recovery after stroke.

The most beneficial time to introduce robotic therapies needs to be evaluated. Therefore, studies in chronic, subacute, and acute populations are all important to perform. We chose to perform this study in subacute subjects instead of chronic subjects primarily to avoid secondary maladaptive changes related to increased passive tissue stiffness and contractures, which are likely to be more severe in the chronic stages after stroke. Furthermore, in the subacute phase, the central nervous system may be more amenable to neural plasticity than in the chronic phase. We elected to use subacute subjects instead of acute subjects for the following reasons:

1. Although testing in the acute phase compared with the subacute phase has theoretical advantages, subjects are often too ill to tolerate additional therapy.

2. In the subacute phase, subjects are typically receiving outpatient therapy a few times a week and may tolerate additional therapy but cannot get it because of the costs involved.

3. Pragmatically, robotic therapy might have its largest effect during subacute treatment.

\section{METHODS}

Subacute subjects were included in the current study if they had a diagnosis of a single cerebrovascular accident (CVA) and were 1 to 5 months post-CVA. Subjects were allowed to continue any outpatient therapies in which they were enrolled at the time of study acceptance. Subjects were excluded from the study if they exhibited any upper-limb joint pain or ROM limitations that would limit their ability to complete the protocols. Subjects with any unstable cardiovascular, orthopedic, or neurological conditions were also excluded. Cognitive impairments were screened with the Folstein Mini-Mental State Examination, and subjects were excluded if they scored $<21$ on the Examination.

Subjects were stratified by the FM score and the cerebral hemisphere in which the stroke occurred and then randomly assigned to one of four treatment groups (one control and three robot). Thirty subjects completed the training and posttreatment evaluations. Over 4 weeks, all groups received 15 one-hour treatment sessions held in the same treatment area and supervised by a single occupational therapist. Thus, all subjects received the same treatment time per session, number of sessions per week, and total number of sessions. Subjects in the three robot groups received 50 min of robot-assisted movement each session while subjects in the control group received $50 \mathrm{~min}$ of conventional treatment. All subjects received 5 min of tone normalization and limb positioning at the beginning and end of each session. We did not inform subjects of the explicit goals of the clinical trial, only that the effectiveness of several treatments was being tested. The local institutional review committee approved all protocols, and we obtained informed consent from all subjects.

The robotic treatment was similar to that used in the earlier chronic study. A core set of 12 targeted reaching movements was used that was identical to the patterns used in the chronic study. Subjects practiced some or all of these movements in each session. The four treatment groups were as follows:

1. The robot-unilateral group $(n=9)$ performed exercises that progressed from the easiest exercise modes (passive) to the most challenging (active-constrained). No bilateral exercise was performed.

2. The robot-bilateral group $(n=5)$ practiced the same 12 reaching movements, but only in bilateral mode. Rhythmic circular movements were also performed. 
3 . The robot-combined group $(n=10)$ spent approximately half the treatment time in the unilateral mode and the other half in the bilateral mode. This group received essentially the same robotic treatment as the earlier chronic subjects.

4. The control group ( $n=6)$ received an equivalent intensity and duration of conventional therapy targeting proximal upper-limb function based on NDT. The procedures used in the control group were identical to those used in the chronic study.

An occupational therapist blinded to group assignments tested all subjects with a battery of clinical evaluations immediately before treatment started, immediately posttreatment, and 6 months after treatment ended. Motor impairment was assessed with the upper-limb portion of the FM [25] and the Motor Status Score (MSS) [27]. We used the Functional Independence Measure (FIM) [28] to measure improvements in basic ADL, and the Motor Power examination to assess strength in the upper limb [29]. This motor power examination scores several joints of the proximal upper limb on a 5-point scale. We used the modified Ashworth scale [30] to test for hypertonia in several upper-limb joints. A higher score on the modified Ashworth scale indicates higher tone, so a lower score indicates lower abnormal tone. In all the other scales, higher scores indicate improvement and reduced impairment.

Baseline differences between groups were evaluated with $t$-tests (continuous and ordinal data) and $\chi^{2}$ tests (categorical data). The motor FM was separated into proximal (shoulder and elbow: 42 points) and distal (hand and wrist: 24 points) portions for statistical analysis. The proximal MSS (shoulder and elbow parts) was separated into a movement scale (46 points) and a synergy scale (20 points) that assesses the ability to suppress abnormal synergies during the attempted movements. A higher score on the MSS synergy scale indicates more isolated movement. Because the FM and the MSS movement scales overlap considerably, the latter was not included in the analysis. The modified Ashworth scale scores for individual joints were grouped into a proximal score (maximum $[\max ]=15$ : shoulder internal rotators, elbow extensors, elbow flexors), and a distal score ( $\max =30$ : pronators, supinators, wrist flexors, wrist extensors, digit flexors, digit extensors). For the FIM, only the self-care and transfer sections were considered $(\max =63)$.

Initially, the randomization procedure provided a uniform number of subjects in each group. However, an interim analysis at the midpoint of the study indicated that statistical difference between the robot-combined and control groups had already been achieved. At that point, a trend indicated the robot-combined and the robotunilateral group gains were different. The study investigators decided to use the remaining resources to further investigate this trend; these two groups were continued while the control and robot-bilateral groups were discontinued. To evaluate our initial hypotheses, we performed two preplanned comparisons using $t$-tests. The first comparison was between gains in the robot-combined and robot-unilateral groups. The assumption of homogeneity of variance was tested with the $F$-test. In cases where this assumption was violated, the Robust Rank-Order test [31] was used. The second comparison was between the robot-combined and the control groups. Because of the small number of control subjects $(n=16)$, the nonparametric Robust Rank-Order test was used.

The data collected in this study were compared with the spontaneous recovery patterns reported by Duncan et al. in a study of 459 stroke subjects [32]. Duncan et al. separated subjects into mild, moderate, and severe stroke categories and charted their recovery patterns on the upper-limb FM. They found that the recovery pattern varied depending on the stroke severity. Our subject pool fell between the moderate and severe stroke profiles reported in their study. Therefore, to estimate the spontaneous recovery profile of our subject pool, we calculated leastsquares exponential fits for the moderate and severe profiles. The parameters of these two equations were linearly interpolated until the recovery profile that intersected the baseline data point of our subject pool was found.

\section{RESULTS}

Thirty subjects completed the treatments and the posttreatment evaluations. Six-month follow-up data were available from 23 subjects. One subject dropped out of the study for reasons unrelated to the study. Table 1 summarizes the baseline characteristics of the subjects. Compared with the robot-unilateral group $(n=9)$, the robot-combined group $(n=10)$ had significantly higher tone in proximal joints (proximal modified Ashworth scale, $p<0.05$ ) and more abnormal synergies (MSS synergy, $p<0.05$ ) at baseline. No significant baseline differences were found between groups in age, weeks post-CVA, sex, side of lesion, or any other clinical evaluations. 
Table 1.

Baseline characteristics of subjects $(n=30)$ in each treatment group for subacute study of Mirror Image Movement Enabler for upper-limb rehabilitation.

\begin{tabular}{|c|c|c|c|c|}
\hline Clinical Scale* & Robot-Combined & Robot-Unilateral & Robot-Bilateral & Control \\
\hline Number of Subjects $(n)$ & 10 & 9 & 5 & 6 \\
\hline Age (yr) & $62.3 \pm 2.8$ & $69.8 \pm 4.0$ & $72.2 \pm 11.7$ & $59.9 \pm 5.5$ \\
\hline Post-CVA (wk) & $13.0 \pm 2.1$ & $10.0 \pm 1.9$ & $6.2 \pm 1.0$ & $10.6 \pm 2.7$ \\
\hline Sex (male/female) & $9 / 1$ & $5 / 4$ & $2 / 3$ & $4 / 2$ \\
\hline Side of Lesion (right/left) & $5 / 5$ & $5 / 4$ & $3 / 2$ & $4 / 2$ \\
\hline Sensation (12) & $10.4 \pm 1.2$ & $10.2 \pm 0.9$ & $8.2 \pm 2.4$ & $11.2 \pm 0.8$ \\
\hline Proximal FM (42) & $16.2 \pm 2.5$ & $23.2 \pm 3.2$ & $24.6 \pm 4.2$ & $21.0 \pm 4.0$ \\
\hline Distal FM (24) & $5.5 \pm 2.4$ & $8.4 \pm 2.2$ & $14.6 \pm 4.4$ & $5.0 \pm 2.5$ \\
\hline MSS Synergy (20) & $4.4 \pm 1.4^{\dagger}$ & $10.3 \pm 1.8$ & $8.4 \pm 3.4$ & $9.0 \pm 2.0$ \\
\hline Motor Power (70) & $30.4 \pm 5.1$ & $38.7 \pm 4.8$ & $40.8 \pm 8.1$ & $39.0 \pm 4.8$ \\
\hline FIM (63) & $48.1 \pm 3.2$ & $45.0 \pm 4.0$ & $51.0 \pm 3.3$ & $52.8 \pm 3.4$ \\
\hline
\end{tabular}

The amount of outside physical or occupational therapy targeting upper-limb function was tabulated. The mean value across groups was $0.71 \pm 0.20 \mathrm{~h} /$ wk during treatment and $0.24 \pm 0.14 \mathrm{~h} / \mathrm{wk}$ during the 6-month follow-up. (All values shown are mean \pm standard error of the mean unless stated otherwise.) Statistical analysis found that the amount of outside therapy between groups differed neither during the treatment period nor during the interval between the end of treatment and the 6-month follow-up.

MIME system automatically recorded the amount of time spent performing movements in the robot. Mean time per session was $43.0 \pm 1.6,36.2 \pm 2.1$, and $42.4 \pm$ $2.0 \mathrm{~min}$ for the robot-combined, robot-unilateral, and robot-bilateral groups respectively. The difference between the robot-combined and robot-unilateral groups was significant $(p<0.05)$. We attribute this difference to the fact that the active-constrained unilateral mode required maximal effort, while the bilateral mode required a submaximal effort. Therefore, during activeconstrained mode, subjects rested more between trials than during the bilateral mode (this rest time is not included in session times listed above). This discrepancy in time spent performing movements in the robot does not compromise the results since the overall session times were strictly controlled to be $1 \mathrm{~h}$ across all subjects.
Table 2 summarizes the gains in clinical outcomes. The first comparison was between robot-combined ( $n=$ $10)$ and control $(n=6)$ groups. When compared with the control group at posttreatment, the robot-combined group had greater gains in the proximal FM $(p<0.05)$ and the MSS synergy scale $(p<0.05)$. However, both of these differences were lost at the 6-month follow-up. Next, we considered the comparison between the robot-combined and robot-unilateral groups $(n=9)$. Significant gains were present in both groups in the proximal FM, distal FM, Motor Power examination, and FIM $(p<0.05)$, but none of these gains differed between groups. Betweengroup comparisons were not performed on the modified Ashworth and MSS synergy scales because of significant baseline differences between these two groups. Withingroup analysis found that the robot-combined group had significant gains on the MSS synergy scale $(p<0.05)$, while the robot-unilateral group had no significant gains on this scale. At the follow-up, the distal FM in the robot-unilateral group had greater improvement compared with the robot-combined group $(p<0.05)$.

The upper-limb FM gains in the robot-combined and robot-unilateral groups exceeded that expected from spontaneous recovery. These two groups were collapsed, and the spontaneous recovery profile that intersected our baseline data point was calculated with data from a previous study that tracked the spontaneous recovery profile 
Table 2.

Average gains in clinical scores of subjects $(n=30)$ in each treatment group from subacute study of Mirror Image Movement Enabler for upperlimb rehabilitation.

\begin{tabular}{|c|c|c|c|c|}
\hline Clinical Scale* & Robot-Combined & Robot-Unilateral & Robot-Bilateral & Control \\
\hline \multicolumn{5}{|l|}{ Posttreatment } \\
\hline Number of Subjects & 10 & 9 & 5 & 6 \\
\hline Proximal Ashworth (15) & $-0.7 \pm 0.7$ & $0.9 \pm 0.6$ & $-0.4 \pm 0.4$ & $-1.3 \pm 0.7$ \\
\hline Proximal FM (42) & $5.3 \pm 1.2^{\dagger}$ & $4.3 \pm 1.4$ & $2.4 \pm 1.5$ & $2.5 \pm 0.6$ \\
\hline Distal FM (24) & $2.3 \pm 0.4$ & $3.6 \pm 1.3$ & $1.4 \pm 0.7$ & $3.3 \pm 1.9$ \\
\hline FIM (63) & $3.1 \pm 1.7$ & $3.7 \pm 1.0$ & $0.8 \pm 0.6$ & $3.2 \pm 1.4$ \\
\hline \multicolumn{5}{|l|}{ 6-Month Follow-Up } \\
\hline Number of Subjects & 6 & 7 & 5 & 5 \\
\hline Proximal Ashworth (15) & $-0.2 \pm 0.5$ & $0.3 \pm 1.1$ & $-2.0 \pm 0.8$ & $0.2 \pm 0.8$ \\
\hline Distal Ashworth (30) & $-0.8 \pm 0.6$ & $-0.6 \pm 0.6$ & $-1.2 \pm 0.8$ & $0.8 \pm 0.7$ \\
\hline FIM (63) & $2.8 \pm 2.4$ & $4.3 \pm 2.7$ & $5.0 \pm 1.4$ & $5.2 \pm 1.7$ \\
\hline $\begin{array}{l}\text { Note: Negative score changes on Ash } \\
{ }^{*} \text { Numbers in parentheses indicate hig } \\
{ }^{\dagger} \text { Significant difference from control; } \\
\text { †Significant difference from unilatera } \\
\text { FIM = Functional Independence Meas }\end{array}$ & $\begin{array}{l}\text { indicate reduced tone. } \mathrm{E} \\
\text { le score. } \\
0.05 \text {. }\end{array}$ & are mean \pm standard errc & mean. & 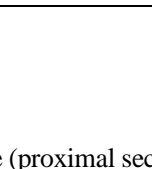 \\
\hline
\end{tabular}

of 459 stroke subjects [32] (see "Methods" section). As depicted in Figure 2, mean gains in the robot-combined and robot-unilateral groups exceeded that expected from spontaneous recovery. Statistical testing compared the FM scores in the robot groups with the corresponding FM levels on the spontaneous recovery curve. Immediately after treatment, a trend $(p<0.1)$ of greater gains was present in the robot groups compared with spontaneous recovery. By the 6-month follow-up, FM scores in the robot groups exceeded those expected from spontaneous recovery $(p<0.05)$.

\section{DISCUSSION}

These results are consistent with the previous study on chronic stroke subjects. In both this and the previous chronic stroke study, proximal FM scores indicated that at posttreatment, robot-combined training group had significantly greater gains than the control group. However, in both studies, gains in robot and control groups were equivalent at the 6-month follow-up. A similar pattern was observed in the MSS synergy score. Thus, the robotcombined treatment is equivalent to conventional treatment in terms of long-term clinical outcomes, but it may accelerate the rate of recovery on some clinical scales.

No significant differences were found between the robot-combined and robot-unilateral treatment on the proximal FM, distal FM, Motor Power examination, and FIM despite that the robot-unilateral subjects spent more time training only the paretic limb. The results also suggest less benefit from bilateral therapy alone, because this group had the smallest gains in the proximal FM, distal FM, Motor Power examination, and FIM. These results have many interpretations. One might argue that because the bilateral mode adds complexity and cost to the robotic device, unilateral modes should be used exclusively without bilateral therapy. Another interpretation is based on the fact that the active unilateral modes require more focused effort and are more fatiguing than the bilateral mode. Combining unilateral and bilateral training might allow extended treatment sessions since the overall effort level is less than in unilateral-only robotic training. Furthermore, the data suggest the bilateral mode may have unique benefits in reducing abnormal synergies. 


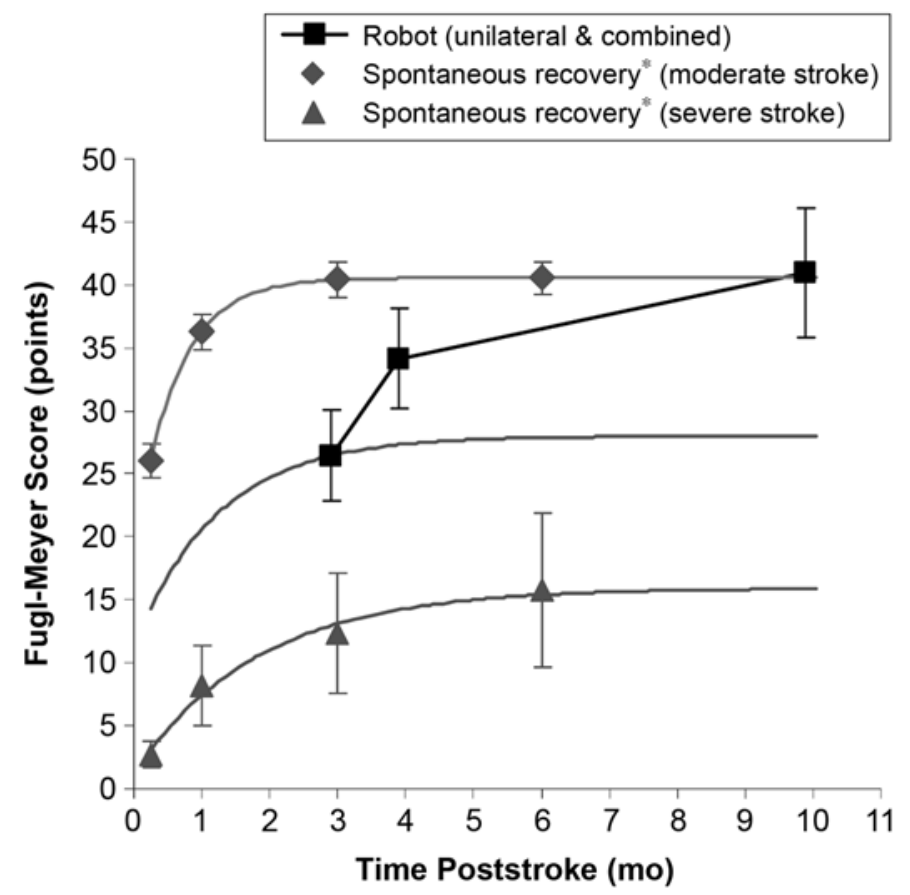

Figure 2.

Gains in upper-limb Fugl-Meyer scores compared with spontaneous recovery patterns. Spontaneous recovery data have been replotted ( and $\boldsymbol{\Delta}$ ) along with exponential fits. Spontaneous recovery curve that intersects baseline point of our subject pool was calculated by interpolating between moderate and severe profiles. *Source: Duncan PW, Lai SM, Keighley J. Defining post-stroke recovery: implications for design and interpretation of drug trials. Neuropharmacology. 2000; 39(5):835-41. [PMID: 10699448]

The robot-combined group showed significant improvement in synergy scores, while the robot-unilateral group showed no improvement. However, this result needs to be reproduced in subject groups that are balanced at baseline in terms of abnormal synergies.

Comparing gains from a treatment with a minimal clinically important difference (MCID) is important. For example, gains might be significant, but the treatment still might not be justified if gains are less than the MCID. Although no standard exists for defining MCID for stroke patients, studies have suggested that the MCID is approximately 10 percent of full scale [33-34]. Based on this assumption, mean gains at posttreatment in the robot-unilateral and robot-combined groups were greater than the MCID in the proximal FM, MSS movement, and Motor Power examination. In the FIM, however, gains were less than the MCID. However, the FIM is a very coarse scale and high scores can be achieved by using only the nonparetic arm. Therefore, the FIM may not be an adequate measure of improved functional use of the paretic upper limb. Thus, the 10 percent assumption might be suspect, and any significant increase in the FIM could be argued to be clinically significant.

Examination of gains in individual subjects suggests the robotic treatment is most effective for subjects in a middle range of motor impairment. Four subjects had gains of greater than 10 points on the proximal FM (two received robot-unilateral treatment and two received robot-combined treatment). These four subjects fell between 15 and 23 inclusive on the proximal FM, while all 30 subjects spanned the range from 7 to 37. Analysis of the data from the previous chronic study also supports this hypothesis. The top three performers in the robot group had pretreatment proximal FM scores between 15 and 23. Thus, in the subacute-chronic phases of recovery, robotic training is apparently most effective in subjects with moderate levels of motor impairment.

One of the most compelling rationales for investigation of robotic therapy is the potential to provide additional effective therapy to patients without increasing the costs of healthcare. If commercially viable robotic devices can be developed that patients can use independently in the home or clinic, our data indicate that training with these robots can be as effective as conventional one-on-one treatment from a therapist. Robotic therapy also increased the rate of recovery compared with conventional treatment, and gains exceeded that which was expected because of spontaneous recovery. These results, coupled with the evidence that higher intensities of conventional therapy produces greater reductions in impairment and disability [35-42], support the use of robots to increase the amount of effective therapy for stroke survivors. One should note that all the chronic subjects and most of the subacute subjects had stopped all formal one-on-one therapy from physical or occupational therapists. Therefore, the additional treatment provided was effective and would not typically have been available to these subjects because of the current structure of the healthcare system. An alternative, equally effective method for delivery of this additional therapy is robotic devices.

\section{CONCLUSIONS}

The precise role of the robot in robotic training has generated considerable debate recently. More specifically, does the presence of the robot provide added benefits compared with equal intensities of nonrobotic training? This is an important question given that robotic devices 
are potentially unsafe, complex, and expensive. This subject was highlighted by the study using the ARM Guide; whereby, subjects who practiced on the robot had gains that were comparable with controls that performed an equal amount of unassisted reaching movements [14]. Determining if robotic assistance improves the effectiveness of movement practice is interesting from a scientific viewpoint, and answering this question would guide refinements in the current robotic devices and also potentially improve conventional hands-on therapy.

However, from a pragmatic viewpoint, it is doubtful that moderately to severely impaired subjects can be motivated to perform highly repetitive, unassisted movements without direct supervision and encouragement from a therapist. In the context of CI therapy, even mildly impaired subjects require physical restraint of the lessimpaired limb and direct therapist supervision to achieve the required amounts of task practice. In moderately to severely impaired populations that require more "handson" treatment, low-cost versions of robotic therapy devices that can be used independently might be the least expensive means of facilitating repetitive movement training. Because of the expense, additional therapy from a human therapist is unavailable to many patients. Thus, if robotic therapy devices can be made safe and costeffective (and we believe that they can), then their commercial development should proceed. We have demonstrated that MIME is at least as effective as an equivalent dose of hands-on therapy in subacute and chronic stroke populations. Until a major breakthrough occurs in the cure of stroke and its sequelae, robot-assisted therapy appears to have an appropriate role in rehabilitation.

In the next 10 years, several lines of work should proceed in parallel:

1. Emphasis should be placed on developing low-cost versions of the robots that have been thoroughly tested with controlled clinical trials.

2. Both this subacute study and the previous chronic study indicated that between the end of treatment and the 6-month follow-up, control subjects had greater gains than robot group subjects. This finding motivates the development of portable devices that can be used as part of a home-based treatment plan following inclinic treatment.

3. Clearly, in the upper limb, the potential benefits of robotic therapy cannot be fully evaluated with devices that address shoulder and elbow movement without treating more distal joints. Additional work is needed to develop devices that integrate wrist and finger function.

4. Other nonrobotic approaches should continue to be investigated and compared with robotic training.

5. Future clinical trials should measure the effects of unsupervised or partially supervised robot therapy. All the studies to date have used a therapist or attendant to supervise the robot treatment. Robots will have the largest impact if patients can be motivated to use them independently. Recent studies have shown that with appropriate technology, the role of the therapist during highly repetitive movement training protocols such as CI therapy can be reduced without loss of treatment effectiveness [43-45].

6. A meta-analysis should be undertaken to establish if the gains from robotic devices are clinically relevant.

\section{ACKNOWLEDGMENTS}

To all subjects who participated in this study, we express our deepest appreciation.

This material was based on work supported by the VA RR\&D merit review grants B2056RA, B2695I, and B2156T.

The authors have declared that no competing interests exist.

\section{REFERENCES}

1. Post-Stroke Rehabilitation Guideline Panel. Post-stroke rehabilitation: Clinical practice guidelines. No. 16. Publication AHCPR 95-0662. Rockville (MD): United States Department of Health and Human Services, Public Health Service, Agency for Health Care Policy and Research; 1995.

2. Dobkin BH. The economic impact of stroke. Neurology. 1995;45(2 Suppl 1):S6-9. [PMID: 7885589]

3. Reinkensmeyer DJ, Emken JL, Cramer SC. Robotics, motor learning, and neurologic recovery. Annu Rev Biomed Eng. 2004;6:497-525. [PMID: 15255778]

4. Taub E, Miller NE, Novack TA, Cook EW 3rd, Fleming WC, Nepomuceno CS, Connell JS, Crago JE. Technique to improve chronic motor deficit after stroke. Arch Phys Med Rehabil. 1993;74(4):347-54. [PMID: 8466415]

5. Mark VW, Taub E. Constraint-induced movement therapy for chronic stroke hemiparesis and other disabilities. Restor Neurol Neurosci. 2004;22(3-5):317-36. [PMID: 15502259]

6. Patton JL, Mussa-Ivaldi FA. Robot-assisted adaptive training: custom force fields for teaching movement patterns. 
IEEE Trans Biomed Eng. 2004;51(4):636-46. [PMID: 15072218$]$

7. Krebs HI, Hogan N, Aisen ML, Volpe BT. Robot-aided neurorehabilitation. IEEE Trans Rehabil Eng. 1998;6(1): 75-87. [PMID: 9535526]

8. Volpe BT, Ferraro M, Lynch D, Christos P, Krol J, Trudell C, Krebs HI, Hogan N. Robotics and other devices in the treatment of patients recovering from stroke. Curr Atheroscler Rep. 2004;6(4):314-19. [PMID: 15191707]

9. Volpe BT, Krebs HI, Hogan N. Robot-aided sensorimotor training in stroke rehabilitation. Adv Neurol. 2003;92:429-33. [PMID: 12760210]

10. Ferraro M, Palazzolo JJ, Krol J, Krebs HI, Hogan N, Volpe BT. Robot-aided sensorimotor arm training improves outcome in patients with chronic stroke. Neurology. 2003; 61(11):1604-7. [PMID: 14663051]

11. Stein J, Krebs HI, Frontera WR, Fasoli SE, Hughes R, Hogan N. Comparison of two techniques of robot-aided upper limb exercise training after stroke. Am J Phys Med Rehabil. 2004;83(9):720-28. [PMID: 15314537]

12. Fasoli SE, Krebs HI, Stein J, Frontera WR, Hogan N. Effects of robotic therapy on motor impairment and recovery in chronic stroke. Arch Phys Med Rehabil. 2003;84(4): 477-82. [PMID: 12690583]

13. Reinkensmeyer DJ, Dewald JP, Rymer WZ. Guidancebased quantification of arm impairment following brain injury: a pilot study. IEEE Trans Rehabil Eng. 1999;7(1): 1-11. [PMID: 10188602]

14. Kahn LE, Lum PS, Reinkensmeyer DJ. Selection of robotic therapy algorithms for the upper extremity in chronic stroke: Insights from MIME and ARM guide results. In: Proceedings of the 8th International Conference on Rehabilitation Robotics; 2003 Apr 23-25; Daejeon, Korea. Daejeon (Korea): Human-Friendly Welfare Robot System Engineering Research Center; 2003. p. 208-10.

15. Lum PS, Reinkensmeyer DJ, Mahoney R, Rymer WZ, Burgar CG. Robotic devices for movement therapy after stroke: current status and challenges to clinical acceptance. Top Stroke Rehabil. 2002;8(4):40-53. [PMID: 14523729]

16. Hesse S, Schmidt H, Werner C, Bardeleben A. Upper and lower extremity robotic devices for rehabilitation and for studying motor control. Curr Opin Neurol. 2003;16(6): 705-10. [PMID: 14624080]

17. Lum PS, Burgar CG, Kenney DE, Van der Loos HF. Quantification of force abnormalities during passive and activeassisted upper-limb reaching movements in post-stroke hemiparesis. IEEE Trans Biomed Eng. 1999;46(6):652-62. [PMID: 10356872]

18. Burgar CG, Lum PS, Shor PC, Machiel Van der Loos HF. Development of robots for rehabilitation therapy: The Palo Alto VA/Stanford experience. J Rehabil Res Dev. 2000; 37(6):663-73. [PMID: 11321002]
19. Lum PS, Lehman SL, Reinkensmeyer DJ. The bimanual lifting rehabilitator: an adaptive machine for therapy of stroke patients. IEEE Trans Rehabil Eng. 1995;3(2):166-74.

20. Lum PS, Reinkensmeyer DJ, Lehman SL. Robotic assist devices for bimanual physical therapy: preliminary experiments. IEEE Trans Rehabil Eng. 1993;1(3):185-91.

21. Luft AR, McCombe-Waller S, Whitall J, Forrester LW, Macko R, Sorkin JD, Schulz JB, Goldberg AP, Hanley DF. Repetitive bilateral arm training and motor cortex activation in chronic stroke: a randomized controlled trial. JAMA. 2004;292(15):1853-61. [PMID: 15494583] Erratum in: JAMA. 2004;292(20):2470.

22. Hesse S, Schulte-Tigges G, Konrad M, Bardeleben A, Werner C. Robot-assisted arm trainer for the passive and active practice of bilateral forearm and wrist movements in hemiparetic subjects. Arch Phys Med Rehabil. 2003;84(6): 915-20. [PMID: 12808550]

23. Lum PS, Burgar CG, Shor PC, Majmundar M, Van der Loos M. Robot-assisted movement training compared with conventional therapy techniques for the rehabilitation of upper-limb motor function after stroke. Arch Phys Med Rehabil. 2002;83(7):952-59. [PMID: 12098155]

24. Bobath B. Adult hemiplegia: Evaluation and treatment. 2nd ed. London (England): William Heinemann Medical Books Ltd; 1978.

25. Fugl-Meyer AR, Jaasko L, Leyman I, Olsson S, Steglind S. The post-stroke hemiplegic patient. 1. A method for evaluation of physical performance. Scand J Rehabil Med. 1975; 7(1):13-31. [PMID: 1135616]

26. Lum PS, Burgar CG, Shor PC. Evidence for improved muscle activation patterns after retraining of reaching movements with the MIME robotic system in subjects with post-stroke hemiparesis. IEEE Trans Neural Syst Rehabil Eng. 2004;12(2):186-94.

27. Ferraro M, Demaio JH, Krol J, Trudell C, Rannekleiv K, Edelstein L, Christos P, Aisen ML, England J, Fasoli SE, Krebs HI, Hogan N, Volpe BT. Assessing the motor status score: A scale for the evaluation of upper limb motor outcomes in patients after stroke. Neurorehabil Neural Repair. 2002;16(3):283-89. [PMID: 12234090]

28. Hamilton BB, Laughlin JA, Fiedler RC, Granger CV. Interrater reliability of the 7-level functional independence measure (FIM). Scand J Rehabil Med. 1994;26(3):115-19. [PMID: 7801060]

29. Gregson JM, Leathley MJ, Moore AP, Smith TL, Sharma AK, Watkins CL. Reliability of measurements of muscle tone and muscle power in stroke patients. Age Ageing. 2000; 29(3):223-28. [PMID: 10855904]

30. Bohannon RW, Smith MB. Interrater reliability of a modified Ashworth scale of muscle spasticity. Phys Ther. 1987; 67(2):206-7. [PMID: 3809245] 
31. Siegel S, Castellan NJ Jr. Nonparametric statistics for the behavioral sciences. 2nd ed. Boston (MA): McGraw-Hill; 1988. p. 137-44.

32. Duncan PW, Lai SM, Keighley J. Defining post-stroke recovery: implications for design and interpretation of drug trials. Neuropharmacology. 2000;39(5):835-41. [PMID: 10699448]

33. Van der Lee JH, Beckerman H, Knol DL, De Vet HC, Bouter LM. Clinimetric properties of the motor activity log for the assessment of arm use in hemiparetic patients. Stroke. 2004;35(6):1410-14. [PMID: 15087552]

34. Van der Lee JH, Wagenaar RC, Lankhorst GJ, Vogelaar TW, Deville WL, Bouter LM. Forced use of the upper extremity in chronic stroke patients: results from a single-blind randomized clinical trial. Stroke. 1999;30(11):2369-75. [PMID: 10548673] Comment in: Stroke. 2000;31(4):986-88.

35. Chen CC, Heinemann AW, Granger CV, Linn RT. Functional gains and therapy intensity during subacute rehabilitation: a study of 20 facilities. Arch Phys Med Rehabil. 2002;83(11):1514-23. [PMID: 12422318$]$

36. Kwakkel G, Wagenaar RC, Twisk JW, Lankhorst GJ, Koetsier JC. Intensity of leg and arm training after primary middle-cerebral-artery stroke: a randomised trial. Lancet. 1999;354(9174):191-96. [PMID: 10421300]

37. Parry RH, Lincoln NB, Vass CD. Effect of severity of arm impairment on response to additional physiotherapy early after stroke. Clin Rehabil. 1999;13(3):187-98. [PMID: 10392645]

38. Bode RK, Heinemann AW, Semik P, Mallinson T. Relative importance of rehabilitation therapy characteristics on functional outcomes for persons with stroke. Stroke. 2004; 35(11):2537-42. [PMID: 15472085]
39. Van Peppen RP, Kwakkel G, Wood-Dauphinee S, Hendriks HJ, Van der Wees PJ, Dekker J. The impact of physical therapy on functional outcomes after stroke: what's the evidence? Clin Rehabil. 2004;18(8):833-62. [PMID: 15609840$]$

40. Kwakkel G, Wagenaar RC, Koelman TW, Lankhorst GJ, Koetsier JC. Effects of intensity of rehabilitation after stroke. a research synthesis. Stroke. 1997;28(8):1550-56. [PMID: 9259747]

41. Ottenbacher KJ, Jannell S. The results of clinical trials in stroke rehabilitation research. Arch Neurol. 1993;50(1):37-44. [PMID: 8418798]

42. Langhorne P, Wagenaar RC, Partridge C. Physiotherapy after stroke: more is better? Physiother Res Int. 1996;1(2): 75-88. [PMID: 9238725]

43. Lum PS, Taub E, Schwandt D, Postman M, Hardin P, Uswatte G. Automated Constraint-Induced Therapy Extension (AutoCITE) for movement deficits after stroke. J Rehabil Res Dev. 2004;41(3A):249-58. [PMID: 15543442]

44. Taub E, Lum PS, Hardin P, Mark VW, Uswatte G. AutoCITE: automated delivery of CI therapy with reduced effort by therapists. Stroke. 2005;36(6):1301-4. [PMID: 15879335]

45. Lum PS, Uswatte G, Taub E, Hardin P, Mark VW. A telerehabilitation approach to delivery of constraint-induced movement therapy. J Rehabil Res Dev. 2006;43(3):391-400. [PMID: 17041824]

Submitted for publication February 14, 2005. Accepted in revised form September 6, 2005. 